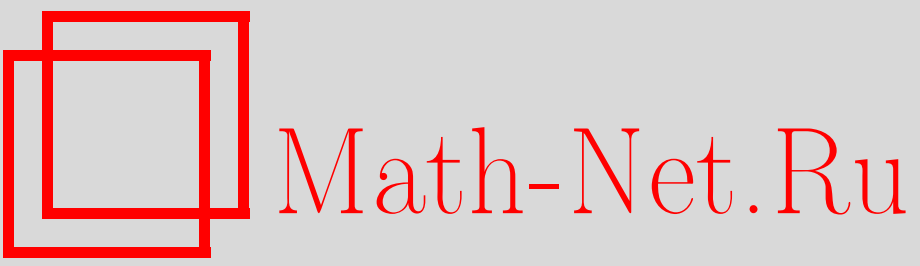

В. И. Качалов, Гладкость по вязкости решений нелинейных дифференциальных уравнений в банаховом пространстве, Итоги науки и техн. Сер. Соврем. мат. и ее прил. Темат. обз., 2021, том 193, 99-103

DOI: https://doi.org/10.36535/0233-6723-2021-193-99-103

Использование Общероссийского математического портала Math-Net.Ru подразумевает, что вы прочитали и согласны с пользовательским соглашением

http://www.mathnet.ru/rus/agreement

Параметры загрузки:

IP : 35.173 .137 .237

26 апреля 2023 г., 12:02:01 


\title{
ГЛАДКОСТЬ ПО ВЯЗКОСТИ РЕШЕНИЙ НЕЛИНЕЙНЫХ ДИФФЕРЕНЦИАЛЬНЫХ УРАВНЕНИЙ В БАНАХОВОМ ПРОСТРАНСТВЕ
}

\author{
(C) 2021 г. \\ В. И. КАЧАЛОВ
}

\begin{abstract}
АннотАция. Аналитические свойства решений дифференциальных уравнений с малым параметром составляют основу аналитической теории возмущений. В случае регулярной теории имеют место теоремы Пуанкаре о разложении или утверждения, вытекающие из концепции аналитического семейства в смысле Като. Когда речь идет о сингулярно возмущенных задачах, то здесь плодотворным является подход, основанный на методе регуляризации С. А. Ломова, центральным понятием которого является понятие псевдоаналитического (псевдоголоморфного) решения, т.е. такого решения, которое представимо в виде сходящегося в обычном смысле ряда по степеням малого параметра.
\end{abstract}

Ключевъе слова: уравнение типа Навье-Стокса, псевдоголоморфное решение, монотонная система норм.

\section{SMOOTHNESS IN THE VISCOSITY OF SOLUTIONS OF NONLINEAR DIFFERENTIAL EQUATIONS IN A BANACH SPACE}

\author{
(C) 2021 V. I. KACHALOV
}

\begin{abstract}
The analytical properties of solutions of differential equations with a small parameter form the basis of analytical perturbation theory. In the case of a regular theory, Poincaré's decomposition theorems or statements that follow from the concept of an analytic family in the sense of Kato hold. For singularly perturbed problems, the approach based on S. A. Lomov's regularization method is useful; the central concept of this method is the concept of a pseudoanalytic (pseudoholomorphic) solution, i.e., a solution, which can be represented in the form of a series converging in the usual sense in powers of a small parameter.
\end{abstract}

Keywords and phrases: Navier-Stokes-type equation, pseudoholomorphic solution, monotone system of norms.

AMS Subject Classification: 34E05, 34K26

1. Введение. Вопрос о гладкости по малому параметру решений дифференциальных уравнений является актуальным как в теории регулярных возмущений, так и в сингулярной теории. В первом случае аналитичность решений начальных задач вытекает либо из теорем Пуанкаре о разложении (см. [5]), либо из понятия подчиненности одного оператора другому (аналитическое семейство в смысле Като (см. [2]). Теория сингулярных возмущений имеет дело в основном с асимптотическими разложениями и поэтому часто называется теорией асимптотического интегрирования (см. $[1,6])$. Установленная С. А. Ломовым возможность обычной сходимости рядов, 
представляющих решения сингулярно возмущенных задач и введенная им концепция псевдоаналитического (псевдоголоморфного) решения легли в основу так называемой аналитической теории сингулярных возмущений (см. [3,7]).

Здесь будет рассмотрено в банаховом пространстве $E$ уравнение, содержащее как линейный, так и билинейный операторы со специальным начальным условием, зависящим от малого параметра.

2. Случай ограниченного билинейного оператора. Будем изучать задачу Коши

$$
u_{t}-A u=B(u, u), \quad t \in[0, T], \quad u(0)=\nu w_{0}(\nu) .
$$

Наложим на данные задачи следующие условия $(\alpha)$ :

(i) замкнутый неограниченный оператор $A$ является инфинитезимальным генератором сильно непрерывной полугруппы $U(t)$ и его область определения $D(A)$ всюду плотна в банаховом пространстве $E$;

(ii) $B(u, v)$ - билинейный ограниченный оператор, определенный на $E \times E$;

(iii) вектор $w_{0}(\nu)$ аналитичен в точке $\nu=0$.

Введем замену $u=\nu w$, тогда получим начальную задачу уже для $w(t)$ :

$$
w_{t}-A w=\nu B(w, w), \quad t \in[0, T], \quad w(0, \nu)=w_{0}(\nu),
$$

решение которой будем искать в виде регулярного ряда по степеням малого параметра:

$$
w(t, \nu)=W_{0}(t, \nu)+\nu W_{1}(t, \nu)+\ldots+\nu^{n} W_{n}(t, \nu)+\ldots
$$

В соответствии с методом неопределенных коэффициентов получим серию начальных задач

$$
\begin{array}{ll}
W_{0, t}-A W_{0}=0, & W_{0}(0, \nu)=w_{0}(\nu), \\
W_{1, t}-A W_{1}=B\left(W_{0}, W_{0}\right), & W_{1}(0, \nu)=0 \\
\ldots \ldots \ldots \ldots \ldots \ldots \ldots \ldots \ldots \ldots & \\
W_{n, t}-A W_{n}=\sum_{k=0}^{n-1} B\left(W_{k}, W_{n-k-1}\right), & W_{n}(0, \nu)=0,
\end{array}
$$

Найдем решения этих задач, пользуясь условием (ii):

$$
\begin{aligned}
W_{0}(t, \nu) & =U(t) w_{0}(\nu) \\
W_{1}(t, \nu) & =\int_{0}^{t} U(t-\tau) B\left(W_{0}(\tau, \nu), W_{0}(\tau, \nu)\right) d \tau \\
\ldots \ldots \ldots \ldots \ldots \ldots \ldots & \ldots \ldots \ldots \\
W_{n}(t, \nu) & =\int_{0}^{t} U(t-\tau)\left[\sum_{k=0}^{n-1} B\left(W_{k}(\tau, \nu), W_{n-k-1}(\tau, \nu)\right)\right] d \tau,
\end{aligned}
$$

Чтобы обеспечить выполнение условия (iii), будем считать, что $\|B(u, v)\| \leqslant b\|u\|\|v\|$, при некотором $b>0$ и любых $u, v \in E$. Пусть $\left\|W_{0}(t, \nu)\right\| \leqslant a$ в некоторой окрестности значения $\nu=0$ при $t \in[0, T]$ и $\|U(t)\| \leqslant q$ на заданном отрезке. Тогда в указанной окрестности $\nu=0$ при всех $t \in[0, T]$ выполнены неравенства

$$
\left\|W_{1}(t, \nu)\right\| \leqslant T q b a^{2}, \quad\left\|W_{2}(t, \nu)\right\| \leqslant T^{2} q^{2} b^{2} a^{3}, \quad \ldots, \quad\left\|W_{n}(t, \nu)\right\| \leqslant T^{n} q^{n} b^{n} a^{n+1}, \quad \ldots,
$$

откуда и следует сходимость ряда (3) в некоторой окрестности значения $\nu=0$.

Сформулируем полученный результат в виде теоремы.

Теорема 1. Если выполнены условия $(\alpha)$, то задача Коши (1) имеет единственное аналитическое в точке $\nu=0$ решение, представимое равномерно сходящимся на отрезке $[0, T]$ рядом. 
Заметим, что единственность следует из способа построения решения, а аналитичность - из аналитичности коэффициентов ряда (3).

В следующем разделе статьи аналогичный результат будет получен для случая неограниченного билинейного оператора.

3. Уравнения типа Навье-Стокса. Снова рассмотрим начальную задачу

$$
u_{t}-A u=B(u, u), \quad t \in[0, T], \quad u(0, \nu)=\nu w_{0}(\nu),
$$

но уже в ситуации, когда билинейный оператор $B(u, v)$ ограничен по первой переменной и является замкнутым неограниченным по второй переменной, с областью определения $D(A) \times D(A)$. Так выглядит уравнение Навье-Стокса, записанное как эволюционное в банаховом пространстве (см. [8]).

После того как сделана замена $u=\nu w$, осуществляется переход к задаче Коши вида (2), для решения которой применяется метод малого параметра и справедливы формулы (4) и (5). Однако оценки вида (6) уже не имеют места из-за неограниченности билинейного оператора. Чтобы доказать утверждение, аналогичное теореме 1 , придется на части области определения $D(B)$ вводить топологию, определяемую счетной системой норм. Пусть $D_{0}$ такое линейное многообразие, содержащееся в $D$, что выполнено следующее условие $(\beta)$ :

(а) на $D_{0}$ можно ввести счетную монотонную систему норм $\|\cdot\|_{k}$ так, что $\|v\|_{1} \leqslant\|v\|_{2} \leqslant \ldots$ для всех $v \in D_{0}$ и из сходимости по каждой из них вытекает сходимость по норме $E$;

(b) $D_{0}$ состоит из таких множеств $Y^{C}(C>0)$, что $\|v\|_{k} \leqslant e^{k C}$ для всех $v \in Y^{C}$ и всех $k \in \mathbb{N}$;

(c) если $u \in Y^{C_{1}}$ и $v \in Y^{C_{2}}$, то $\|B(u, v)\|_{k} \leqslant C_{2} e^{k\left(C_{1}+C_{2}\right)}$;

(d) оператор $U(t)$ равномерно по $t \in[0, T]$ ограничен в счетно-нормированном пространстве $D_{0}$, т.е. найдется такое $q>0$, что $\|U(t) v\|_{k} \leqslant q\|v\|_{k}$ для всех $v \in D_{0}$ и $k \in \mathbb{N}$.

Теорема 2. Если выполнены условия $(\beta)$ и $w_{0}(\nu) \in D_{0}$ при $0 \leqslant \nu \leqslant \nu_{0}$, то задача Коши (7) имеет аналитическое в точке $\nu=0$ решение.

Доказательство. Будем считать, не ограничивая общности, что $q=1$. Заметим, что неравенство в условии (с) можно переписать в виде

$$
\|B(u, v)\|_{k} \leqslant\left. e^{C_{1} x}\left(\partial_{x} e^{C_{2} c}\right)\right|_{x=k} .
$$

Определим функции $P_{n}(k, t)$, мажорирующие $\left\|W_{n}(t, \nu)\right\|_{k}$ рекуррентным образом:

$$
\begin{aligned}
& P_{0}(k, t)=\left.e^{C x}\right|_{x=k}, \\
& P_{1}(k, t)=\left.\int_{0}^{t} P_{0} P_{0, x} d \tau\right|_{x=k}=\left.C t e^{2 C x}\right|_{x=k}, \\
& P_{2}(k, t)=\left.\int_{0}^{t}\left(P_{0} P_{1, x}+P_{1} P_{0, x}\right) d \tau\right|_{x=k}=\left.\frac{3}{2} C^{2} t^{2} e^{3 C x}\right|_{x=k} \\
& P_{n}(k, t)=\left.\int_{0}^{t}\left(P_{0} P_{n-1, x}+P_{1} P_{n-2, x}+\ldots+P_{n-1} P_{0, x}\right) d \tau\right|_{x=k}=\left.\frac{(n+1)^{n-1}}{n !} C^{n} t^{n} e^{(n+1) C x}\right|_{x=k},
\end{aligned}
$$

Эти формулы можно доказать методом математической индукции, а так как ряд

$$
\sum_{k=0}^{\infty} \nu^{n} P_{n}(k, T)
$$

при каждом натуральном $k$ сходится в некоторой окрестности значения $\nu=0$, то и теорему 2 можно считать доказанной. 
Замечание. В случае, если и оператор $A$ зависит от малого параметра $\nu$, то коэффициенты $W_{n}(t, \nu)$ ряда вида (3) могут зависеть от $\nu$ нерегулярным образом. Утверждение теоремы 2 все равно будет верно, поскольку оценки для $\left\|W_{n}(t, \nu)\right\|$, произведенные с помощью формулы (8), не зависят от $\nu$, и решение $w(t, \nu)$ уже будет псевдоаналитическим (псевдоголоморфным).

4. Пример. Сначала приведем утверждение о гладкости по $\nu$ решения задачи Коши в $\mathbb{R}^{m}$ для уравнения диффузии

$$
u_{t}=\nu \Delta u+f(t, x), \quad u(0, x)=\varphi(x)
$$

где $x=\left(x_{1}, \ldots, x_{m}\right), \Delta=\partial_{x_{1}}^{2}+\ldots+\partial_{x_{m}}^{2}$.

Теорема 3. Если функции $f(t, x)$ и $\varphi(x)$ допускают аналитические продолжсения на $\mathbb{C}^{m}$, которые являются целыми функциями порядка $0<\rho \leqslant 2$, т.е.

$$
|f(t, z)| \leqslant M_{0}(t) e^{|z|^{\rho}}, \quad|\varphi(z)| \leqslant M_{1} e^{|z|^{\rho}}
$$

для всех $z \in \mathbb{C}^{m}$, то для любого $T>0$ в полосе $[0, T] \times \mathbb{R}^{m}$ существует единственное аналитическое в точке $\nu=0$ решение задачи (9), $\kappa$ тому же принадлежащее тихоновскому классу корректности параболических задач.

Рассмотрим в $\mathbb{R}^{2}$ задачу Коши

$$
\begin{cases}u_{t}-\nu\left(u_{x x}+u_{y y}\right)=-u u_{x}-v u_{y}, & \left.u\right|_{t=0}=\nu \varphi(x, y, \nu), \\ v_{t}-\nu\left(v_{x x}+v_{y y}\right)=-u v_{x}-v v_{y}, & \left.v\right|_{t=0}=\nu \psi(x, y, \nu) .\end{cases}
$$

Сделаем замену $u=\nu \widetilde{u}, v=\nu \widetilde{v}$, тогда

$$
\begin{cases}\widetilde{u}_{t}-\nu\left(\widetilde{u}_{x x}+\widetilde{u}_{y y}\right)=-\nu\left(\widetilde{u} \widetilde{u}_{x}+\widetilde{v} \widetilde{u}_{y}\right), & \left.\widetilde{u}\right|_{t=0}=\varphi(x, y, \nu), \\ \widetilde{v}_{t}-\nu\left(\widetilde{v}_{x x}+\widetilde{v}_{y y}\right)=-\nu\left(\widetilde{u} \widetilde{v}_{x}+\widetilde{v} \widetilde{v}_{y}\right), & \left.\widetilde{v}\right|_{t=0}=\psi(x, y, \nu) .\end{cases}
$$

Решение системы (11) будем искать в виде рядов по степеням вязкости:

$$
\widetilde{u}=\widetilde{u}_{0}+\nu \widetilde{u}_{1}+\ldots, \quad \widetilde{v}=\widetilde{v}_{0}+\nu \widetilde{v}_{1}+\ldots
$$

Пусть продолжения $\varphi\left(z_{1}, z_{2}, \nu\right)$ и $\psi\left(z_{1}, z_{2}, \nu\right)$ аналитичны в точке $\nu=0$ и являются функциями экспоненциального типа, т.е. существует такое $C>0$, что

$$
\left|\varphi\left(z_{1}, z_{2}, \nu\right)\right| \leqslant e^{C\left(\left|z_{1}\right|+\left|z_{2}\right|\right)}, \quad\left|\psi\left(z_{1}, z_{2}, \nu\right)\right| \leqslant e^{C\left(\left|z_{1}\right|+\left|z_{2}\right|\right)} \quad \forall z_{1}, z_{2} \in \mathbb{C}^{2} .
$$

Члены $\widetilde{u}_{0}$ и $\widetilde{v}_{0}$ найдем без использования формулы Пуассона, применив к уравнениям

$$
\begin{array}{ll}
\widetilde{u}_{0, t}=\nu \Delta \widetilde{u}_{0}, & \left.\widetilde{u}_{0}\right|_{t=0}=\varphi(x, y, \nu), \\
\widetilde{v}_{0, t}=\nu \Delta \widetilde{v}_{0}, & \left.\widetilde{v}_{0}\right|_{t=0}=\psi(x, y, \nu)
\end{array}
$$

теорему 3.

Функции $\widetilde{u}_{0}(t, x, y, \nu)$ и $\widetilde{v}_{0}(t, x, y, \nu)$ будут аналитическими в точке $\nu=0$ в каждой полосе $[0, T] \times \mathbb{R}^{2}$ и принадлежать тому же экспоненциальному типу, что и начальные функции.

Рассмотрим пространство $E=C\left(\mathbb{R}^{2}\right)$ с нормой

$$
\|g\|=\sup _{\mathbb{R}^{2}}|g(x, y)|
$$

и пусть $D_{0}$ состоит из целых функций экспоненциального типа с системой норм

$$
\|g\|_{k}=\max _{\left|z_{1}\right|,\left|z_{2}\right| \leqslant k}\left|g\left(z_{1}, z_{2}\right)\right|,
$$

а билинейный оператор $B$ определяется правой частью системы (10). Для определения $\widetilde{u}_{1}$ и $\widetilde{v}_{1}$ имеем систему

$$
\begin{cases}\widetilde{u}_{1, t}=\nu \Delta \widetilde{u}_{1}-\widetilde{u}_{0} \widetilde{u}_{0, x}-\widetilde{v}_{0} \widetilde{u}_{0, y}, & \left.\widetilde{u}_{1}\right|_{t=0}=0, \\ \widetilde{v}_{1, t}=\nu \Delta \widetilde{v}_{1}-\widetilde{u}_{0} \widetilde{v}_{0, x}-\widetilde{v}_{0} \widetilde{v}_{0, y}, & \left.\widetilde{v}_{1}\right|_{t=0}=0,\end{cases}
$$


которую решим с помощью формулы Пуассона (в скобках аргументы):

$$
\begin{aligned}
& \widetilde{u}_{1}(t, x, y, \nu)=\frac{1}{\pi} \int_{0}^{t} d \tau \int_{-\infty}^{+\infty}\left[-\widetilde{u}_{0} \widetilde{u}_{0, x}-\widetilde{v}_{0} \widetilde{u}_{0, y}\right](\tau, x+2 \sqrt{\nu(t-\tau)} \xi, y+2 \sqrt{\nu(t-\tau)} \eta) e^{-\xi^{2}-\eta^{2}} d \xi d \tau, \\
& \widetilde{v}_{1}(t, x, y, \nu)=\frac{1}{\pi} \int_{0}^{t} d \tau \int_{-\infty}^{+\infty}\left[-\widetilde{u}_{0} \widetilde{v}_{0, x}-\widetilde{v}_{0} \widetilde{v}_{0, y}\right](\tau, x+2 \sqrt{\nu(t-\tau)} \xi, y+2 \sqrt{\nu(t-\tau)} \eta) e^{-\xi^{2}-\eta^{2}} d \xi d \tau,
\end{aligned}
$$

и т. д.

Видно, что $\widetilde{u}_{1}$ и $\widetilde{v}_{1}$ не зависят от вязкости регулярным образом, следовательно, построенное таким образом решение будет псевдоголоморфным в точке $\nu=0$.

5. Заключение. Весьма важным является изучение аналитических свойств решений и других классов нелинейных дифференциальных уравнений в банаховых пространствах, содержащих малый параметр. Итогом этой работы должно стать построение аналитической теории возмущений, охватывающей широкий спектр как регулярных, так и сингулярных задач.

\section{СПИСОК ЛИТЕРАТУРЫ}

1. Васильева А. Б., Бутузов В. Ф. Асимптотическое разложение решений сингулярно возмущенных задач. - М.: Наука, 1973.

2. Като T. Теория возмущений линейных операторов. - М.: Мир, 1972.

3. Качалов В. И. О голоморфной регуляризации сингулярно возмущенных систем дифференциальных уравнений// Ж. вычисл. мат. мат. физ. - 2017. -57 , № 4. - С. $64-71$.

4. Качалов В. И., Федоров Ю. С. О методе малого параметра в нелинейной математической физике// Сиб. электрон. мат. изв. - 2018. - 15. - С. 1680-1686.

5. Коддингтон Э. А., Левинсон Н. Теория обыкновенных дифференциальных уравнений. - М.: ИЛ, 1958.

6. Ломов С. А. Введение в общую теорию сингулярных возмущений. - М.: Наука, 1981.

7. Ломов С. А., Ломов И. С. Основы математической теории пограничного слоя. - М.: Изд-во МГУ, 2011.

8. Рихтмайер Р. Принципы современной математической физики. Т. ІІ. - М.: Мир, 1984.

Качалов Василий Иванович

Национальный исследовательский университет «МЭИ», Москва

E-mail: vikachalov@rambler.ru 